\title{
New opportunities with quantification of protein therapeutics by LC-MS
}

\author{
Hendrik Neubert*,1 \& Keyang $\mathrm{Xu}^{2}$ \\ ${ }^{1}$ Pfizer Inc, 1 Burtt Road, Andover, MA 01810, USA \\ ${ }^{2}$ Genentech Inc., 1 DNA Way, San Francisco, CA 94080, USA \\ *Author for correspondence: Hendrik.Neubert@pfizer.com
}

First draft submitted: 25 May 2018; Accepted for publication: 5 June 2018; Published online: 17 July 2018

Keywords: bottom-up • digestion $\bullet$ HRMS $\bullet$ immunoaffinity $\bullet$ LC-MS $\bullet$ protein quantification $\bullet$ protein therapeutics

We are pleased to be guest editors for this special focus issue of Bioanalysis devoted to quantification of protein therapeutics by LC-MS. Starting in the earlier part of the last decade, bioanalytical scientists in the biopharmaceutical and biotechnology industry as well as academic institutions began exploring the use of MS (mass spectrometry) to quantify protein therapeutics. Motivations igniting and fueling this development included the significant increase in the number and complexity of protein therapeutics being developed, the need for novel bioanalytical assays and tools for new modalities where no suitable solutions existed previously and the growing concurrent experience with protein biomarker quantification by LC-MS.

Now, over a decade later, LC-MS has become an established technology and in some application areas even a preferred platform, for quantification of protein therapeutics. Practically, most if not all protein therapeutics could be measured by LC-MS. However, the field has been focusing on identifying those bioanalytical challenges amenable to this novel approach that would yield the best return on investment. This may differ from lab-to-lab depending on expertise, infrastructure and of course the specific bioanalytical question at hand. As illustrated in this special focus issue of Bioanalysis, it is exciting to observe that the relevant expertise as well as the sample preparation and MS technology continue to advance at a significant pace. Questions such as 'what can be done by LC-MS?', 'what should be done by LC-MS?' or 'what needs to be done by LC-MS?' get constantly revisited as the field advances and the new opportunities surface. Presented by key experts, this themed issue highlights the recent progress in this field and how a variety of bioanalytical challenges are overcome to pave the way for an increasing practical applications of protein therapeutics quantification by LC-MS.

Currently, all sensitive LC-MS assays for protein therapeutics, as well as for protein biomarkers, require some type of analyte enrichment. In this issue, Qu et al. discuss various enrichment strategies that can be chosen based on the intended use of the assay and the desired analytical performance criteria. Importantly, enrichment techniques can also be creatively combined to further enhance assay performance where needed [1]. One particularly powerful and routinely demonstrated form of enrichment is immunoaffinity capture of the protein or peptide analyte. In their perspective, Zhao et al. discuss critical considerations for the implementation of immunoaffinity enrichment strategies. They highlight the criticality of careful reagent generation, selection and characterization. Furthermore, designing the appropriate immunoaffinity assay format is important to develop a reliable bioanalytical assay [2].

Presently, protein quantification by LC-MS usually involves a bottom-up approach in most cases. Such process derives one or more signature peptides from the analyte of interest via proteolytic digestion, which subsequently serve as surrogate(s) for quantification. Sucharski et al. describe a bottom-up LC-MS approach to quantify an IgG1 $\mathrm{mAb}$ therapeutic in human serum. A surrogate tryptic CDR peptide is enriched online using a rabbit polyclonal antipeptide antibody. This LC-MS assay not only generates the results comparable with that obtained previously using a target-based ELISA, but also effectively addresses matrix interferences experienced in the LBA format [3].

Although trypsin digestion usually yields suitable surrogate peptides, increasingly there are situations where that is not the case, especially as we encounter more diverse and complex protein therapeutic modalities. Alternative enzymes or enzyme cocktails have been explored to obtain surrogate peptides that can afford favorable ionization and fragmentation for higher sensitivity and selectivity. Hansen $e t$ al. share an interesting case study where an alternative 
enzyme, Glu-C is used to produce unique surrogate peptides from a bispecific IgG1 therapeutic antibody in preclinical matrices [4].

Over the years, tandem MS with unit mass resolution afforded by the triple quadrupole MS has been the dominant detection means for protein LC-MS assays. However, Bults et al. argue that selectivity and sensitivity of the assay can potentially be compromised due to interferences from endogenous peptides generated from the matrix proteome, which share strong similarity to the surrogate peptide and can be difficult for a triple quadrupole MS to resolve. The authors report a quadrupole time-of-flight MS approach to enhance the measurement of biopharmaceutical somatropin in rat plasma. They anticipate that HRMS (high-resolution mass spectrometry) will become more prevalent for protein quantification in the near future [5].

Even though bottom-up LC-MS assays have been most frequently developed and implemented for protein therapeutics, the importance of characterization and quantification of protein analytes at the intact level has gained increasing attention in the recent years. Using HRMS, the top-down approach, under denatured or native conditions, may allow the detection of both intact therapeutics and relevant modifications/catabolites in circulation, which can ultimately gain further insights into understanding efficacy or safety. Zhang et al. present a method using top-down quadrupole time-of-flight to measure an intact, native antibody isolated from incurred biological samples. They suggest that top-down native MS may serve as a complement or alternative to ligand binding assay or bottom-up LC-MS for protein quantification [6]. Meanwhile, Qiu et al. present a comprehensive evaluation on how to perform intact protein quantitative analysis using two different data processing strategies, deconvoluted mass spectra or extracted ion chromatogram. Both approaches correlate well with each other, as well as show a good agreement with the surrogate peptide analysis [7]. However, both groups acknowledge that a breakthrough in sensitivity is critical for broader applications of intact MS analysis in protein drug development.

Recent progress in immunoaffinity (or hybrid) LC-MS has undoubtedly asserted its utility in quantifying protein therapeutics in biological matrices. With an increasing demand for its application in the regulated environment, guiding principles for validation of hybrid LC-MS assays have been actively discussed and proposed. An editorial by Duggan summarizes various experiments to be included during validation to ensure method reliability. The reader is reminded that as LC-MS assays become more sensitive, potential ligand-binding interferences should be carefully assessed, as well as life-cycle management of the critical reagents for analyte enrichment [8].

So are we ready to declare hybrid LC-MS assays as the next big thing? The editorial by Booth and Furmanski from the US FDA provides their perspective. The authors acknowledge that preliminary hybrid assay results reported so far seem to demonstrate the robustness in assay performance. However, they also raise a few questions regarding the complexity of the hybrid LC-MS approach due to multiple steps employed. These questions intend to prompt continued and timely dialog between scientists in drug development field and the regulators. The authors expect that the agency will continue to work with the industry to implement this new technology in the future [9].

We have highlighted content in this special focus Issue dedicated to progress and innovation in the quantification of protein therapeutics by LC-MS. We hope and anticipate that the discussions and research shown herein will spark new ideas and foster more collaboration to further advance this important area of bioanalysis. The aim is to put increasingly capable bioanalytical tools in the hands of researchers and drug developers, ultimately to help accelerate the development of protein therapeutics for patients.

Financial \& competing interests disclosure

$\mathrm{H}$ Neubert is an employee of, and holds stock or stock options in Pfizer. K Xu is an employee of, and holds stock or stock options in Genentech/Roche. The authors have no other relevant affiliations or financial involvement with any organization or entity with a financial interest in or financial conflict with the subject matter or materials discussed in the manuscript apart from those disclosed. No writing assistance was utilized in the production of this manuscript.

\section{References}

1. Pu J, An B, Vazvaei F, Qu J. Enrichment of protein therapeutics and biomarkers for LC-MS quantification. Bioanalysis 10(13), 979-982 (2018).

2. Zhao Y, Gu H, Zheng H, Zeng J. Critical considerations for immunocapture enrichment LC-MS bioanalysis of protein therapeutics and biomarkers. Bioanalysis 10(13), 987-995 (2018).

3. Sucharski FK, Meier S, Miess C et al. Development of an automated, interference-free, 2D-LC-MS/MS assay for quantification of a therapeutic mAb in human sera. Bioanalysis 10(13), 1023-1037 (2018). 
4. Hansen K, Szarka S, Escoffier E et al. Glu-C: an alternative digestive enzyme for the quantitative LC-MS/MS analysis of an IgG-based antibody biotherapeutic. Bioanalysis 10(13), 997-1007 (2018).

5. Bults P, Meints M, Sonesson A, Knutsson M, Bischoff R, van de Merbel NC. Improving selectivity and sensitivity of protein quantitation by LC-HR-MS/MS: determination of somatropin in rat plasma. Bioanalysis 10(13), 1009-1021 (2018).

6. Zhang L, Vasicek LA, Bateman K, Hsieh S, Zhang S, Henion J. Top-down LC/MS quantitation of intact denatured and native monoclonal antibodies in biological samples. Bioanalysis 10(13), 1039-1054 (2018).

7. Qiu X, Kang L, Case M, Weng N, Jian W. Quantitation of intact monoclonal antibody in biological samples: comparison of different data processing strategies. Bioanalysis 10(13), 1055-1067 (2018).

8. Duggan JX. Progress in high-sensitivity hybrid LC-MS/MS methods for the bioanalysis of protein drugs and performance tests for their validation. Bioanalysis 10(13), 983-986 (2018).

9. Booth BP, Furmanski B. Hybrid assays: the next big thing. Bioanalysis 10(13), 975-977 (2018). 
